Supporting Information for:

\title{
Surface Roughness Modulates Diffusion and Fibrillation of Amyloid- $\beta$ Peptide
}

\author{
Khurram Shezad ${ }^{\dagger}$, Kejun Zhang ${ }^{\dagger}$, Mubashir Hussain ${ }^{\dagger}$, Hai Dong ${ }^{\dagger}$, Chuanxin $\mathrm{He}^{\ddagger}$, \\ Xiangjun Gong ${ }^{\S}$, Xiaolin $\mathrm{Xie}^{\dagger}$, Jintao $\mathrm{Zhu}^{\dagger}{ }^{\dagger}$, , Lei Shen ${ }^{\dagger, *}$
}

†School of Chemistry and Chemical Engineering, Huazhong University of Science and Technology, Wuhan 430074, China

†College of Chemistry and Environmental Engineering, Shenzhen University, Shenzhen 518060, China

$\S$ School of Materials Science and Engineering, South China University of Technology, Guangzhou 510640, China

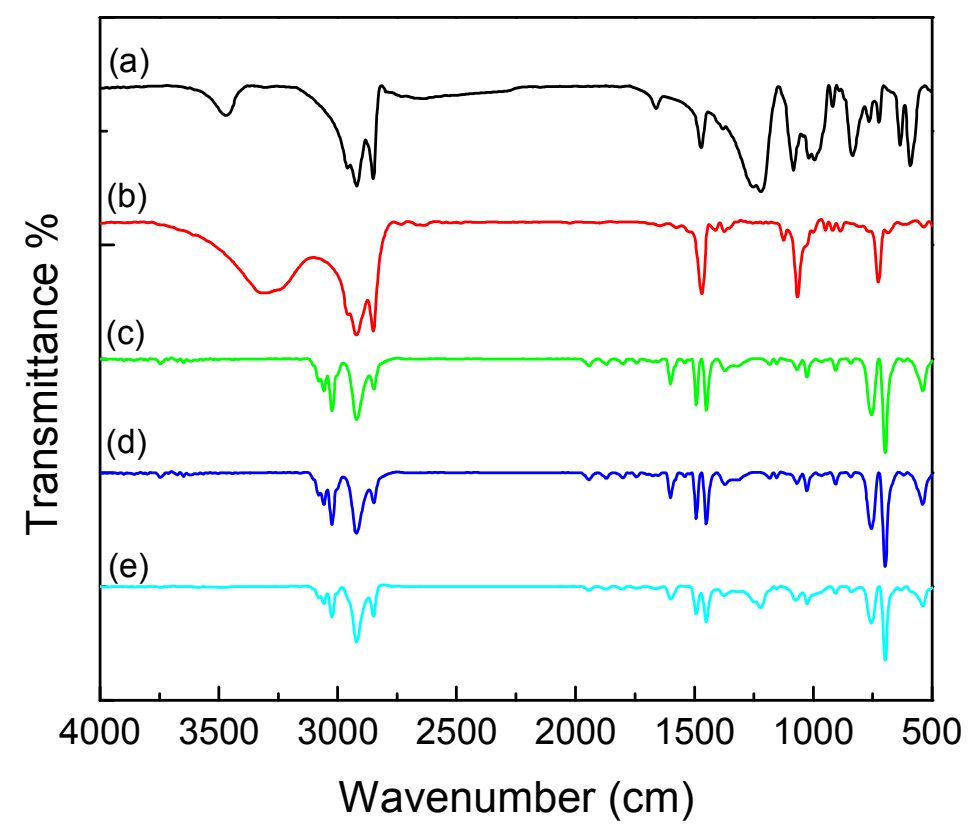

Figure S1. IR spectra of (a) neat SDS, (b) neat HD, (c) smooth PS microparticle, (d) slightly rough PS microparticle and (e) highly textured PS microparticle. Clearly, SDS has been removed completely from the surface of PS particles after the dialysis treatment. 


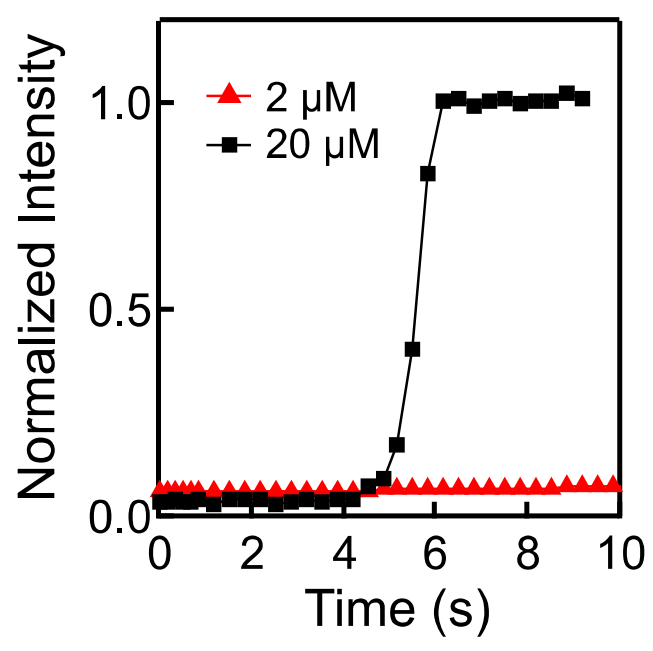

Figure S2. Plots showing the fibrillation kinetics of $\mathrm{A} \beta_{42}$ at different concentrations $(\boldsymbol{\Lambda}, 2 \mu \mathrm{M} ; \mathbf{m}$, $20 \mu \mathrm{M})$ at $37^{\circ} \mathrm{C}$ monitored through ThT fluorescence method without any adding surfaces. There is no obvious increase of fluorescence signal during our experimental time window for $A \beta$ solution at $2 \mu \mathrm{M}$, which indicates that fibrils indeed do not form in solution phase at this condition. 
1 hour
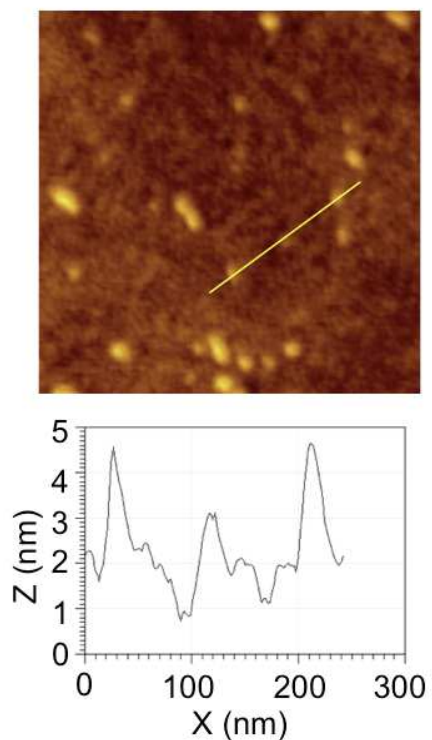

3 hour
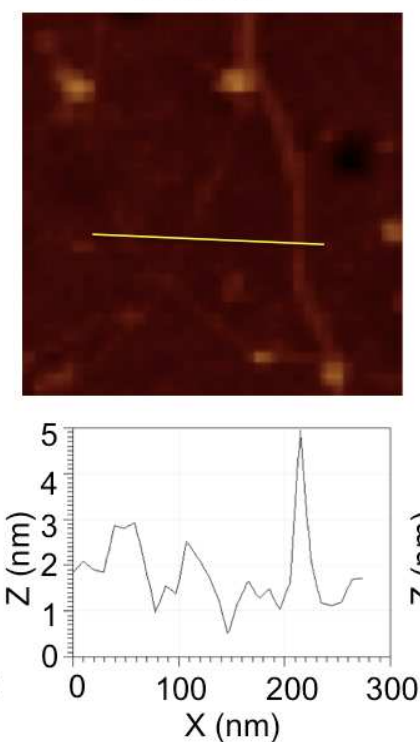

6 hour
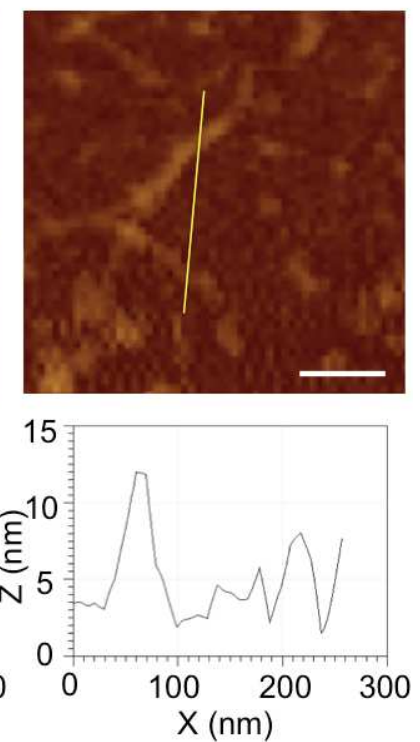

Figure S3. AFM images taken in the air for the smooth PS surfaces after they have been incubated in $\mathrm{A} \beta_{42}$ peptide solution ( $2 \mu \mathrm{M}$ in PBS) at $37{ }^{\circ} \mathrm{C}$ for 1,3 , and 6 hours. The scale bar is $100 \mathrm{~nm}$ for all panels. The corresponding height profiles are shown below each image. As shown by above AFM images, particle-like structures are present on smooth surface after 1 hour of incubation. These particles have heights ranging from $\sim 1$ to $\sim 2.5 \mathrm{~nm}$, which indicates that these particles represent different species of peptide oligomers. After 3 hours of incubation, long fibrils with a height range from $\sim 1$ to $\sim 3 \mathrm{~nm}$ readily grow on the smooth surface through addition of monomers at the edge of oligomers. This value is in agreement with the single-strand long fibrils with height of 1.5-3 nm determined for fibrillation of A $\beta 42$ on graphite surface (Langmuir 2012, 28, 6595-6605). After 6 hours of incubation, thicker fibrils with height of 5-10 nm are observed. These mature fibrils were attributed to the lateral association and intertwine of two or more protofibrils together. In addition to the mature fibrils, there are still large numbers of amorphous aggregates present on the surface. 

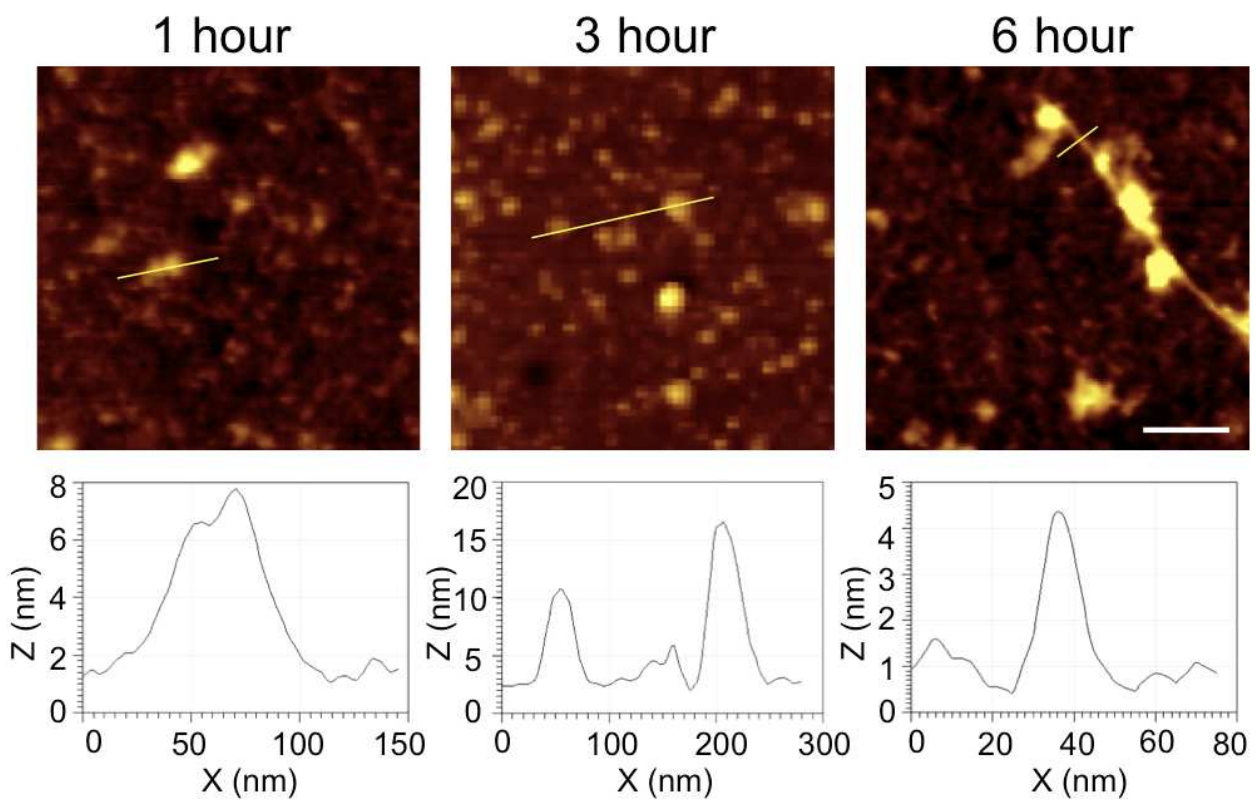

Figure S4. AFM images taken in the air for the slightly rough PS surfaces after they have been incubated in $\mathrm{A} \beta_{42}$ peptide solution ( $2 \mu \mathrm{M}$ in PBS) at $37{ }^{\circ} \mathrm{C}$ for 1,3 , and 6 hours. The scale bar is $100 \mathrm{~nm}$ for all panels. The corresponding height profiles are shown below each image. As shown by above AFM images, oligomers are again found as the primary species of aggregation from the beginning stage on slightly rough surface. The oligomers are larger than those present on smooth surface. With increasing incubation time from 1 to 3 hours, the density of oligomers on the surface increases together with numbers of larger particle-like aggregates. After 6 hours of incubation, single-strand short fibrils with height of $\sim 3 \mathrm{~nm}$, not elongated ones, are observed on the surface. 

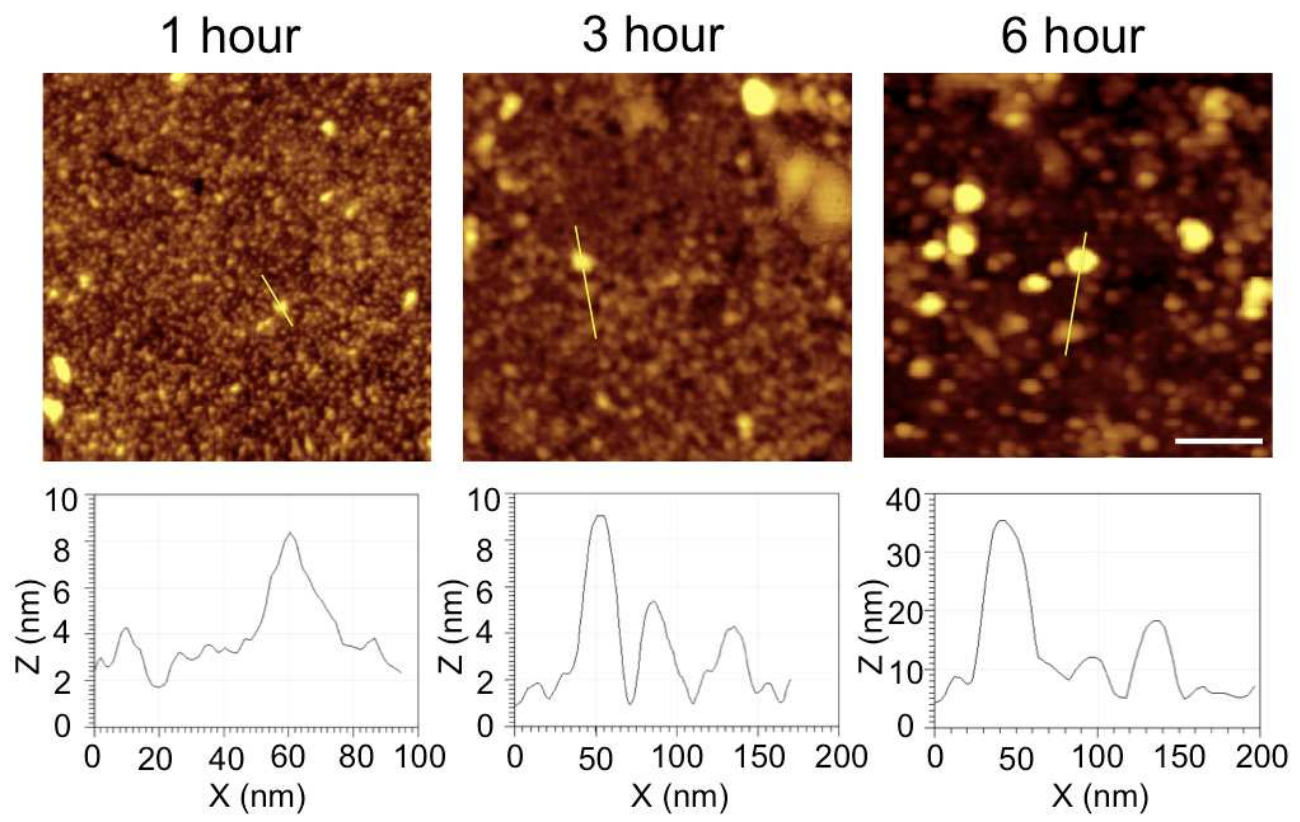

Figure S5. AFM images taken in the air for the highly rough PS surfaces after they have been incubated in $\mathrm{A} \beta_{42}$ peptide solution ( $2 \mu \mathrm{M}$ in PBS) at $37{ }^{\circ} \mathrm{C}$ for 1,3 , and 6 hours. The scale bar is $100 \mathrm{~nm}$ for all panels. The corresponding height profiles are shown below each image. As shown by above AFM images, no fibrillation is observed within 6 hours of incubation on highly rough surface, and oligomers and amorphous aggregates represent the only self-assembly of peptide monomers. The size and density of aggregates increase with increasing the incubation time. The density of aggregates on highly rough surface is higher than that on smooth and slightly rough surfaces at the same time window. 

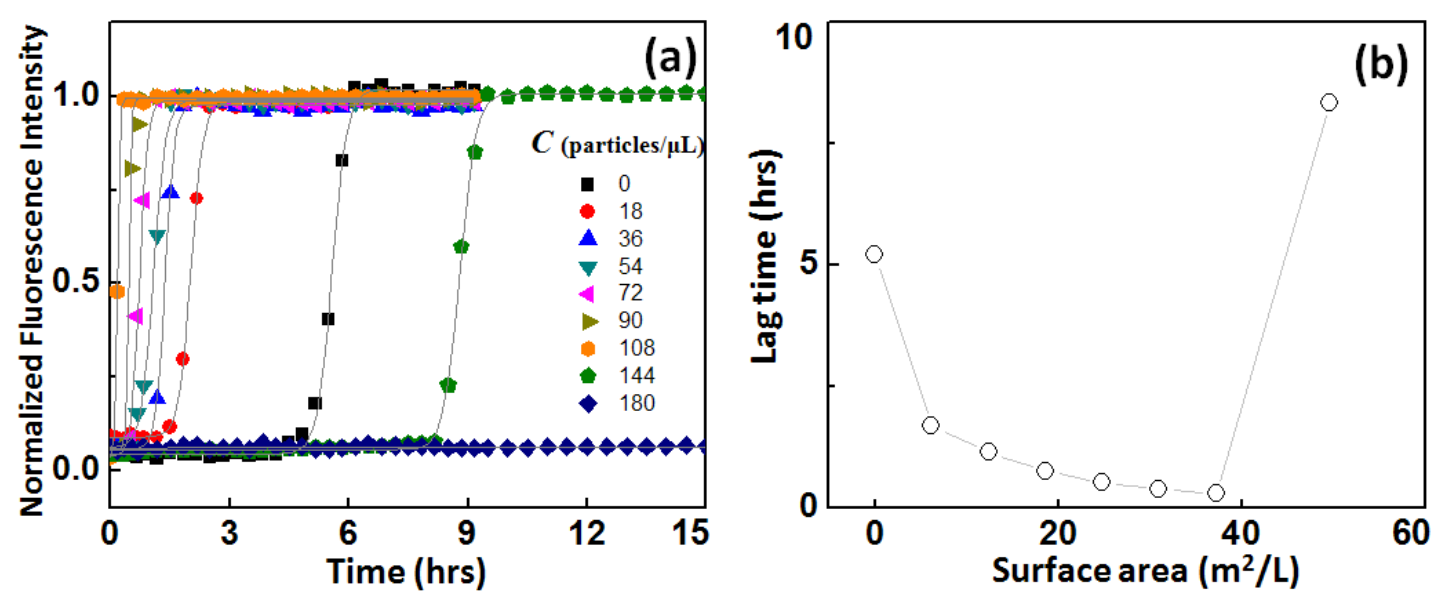

Figure S6. (a) Plots show the fibrillation kinetics of $\mathrm{A} \beta_{42}(20 \mu \mathrm{M})$ at $37^{\circ} \mathrm{C}$ monitored through ThT fluorescence method for different number concentrations of smooth PS microparticles. Lines represent the best fit of an empirical sigmoidal equation based on Eq. 1. (b) Plots show the lag time as a function of particle surface area in solution based on Eq. 2.

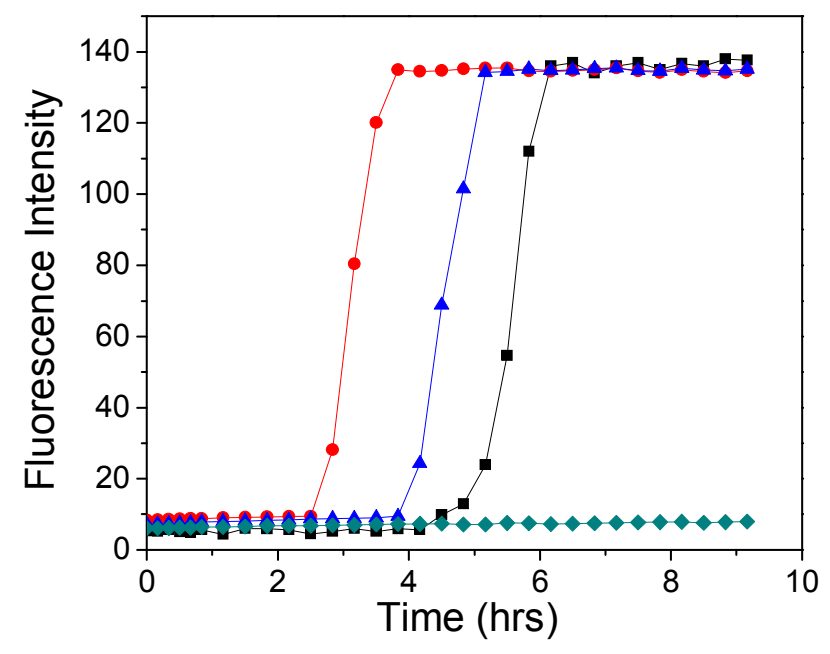

Figure S7. Plots showing the fibrillation kinetics of $\mathrm{A} \beta_{42}(20 \mu \mathrm{M})$ at $37^{\circ} \mathrm{C}$ monitored through ThT fluorescence method in the presence of $(\boldsymbol{O})$ smooth, $(\boldsymbol{\Delta})$ slightly rough, $(\boldsymbol{\nabla})$ highly textured PS particles and in the absence of PS particles $(\boldsymbol{\square})$. The number concentration of the PS particles is 5 particles $/ \mu \mathrm{L}$.

Control experiment of $\mathrm{A} \beta_{42}$ fibrillation initiated by smooth particles: Different concentrations of $\mathrm{A} \beta_{42}$ with $200 \mu \mathrm{M}$ ThT dye was incubated in the absence or presence of microparticles for each fluorescence experiment. Fig. S6a shows $\mathrm{A} \beta_{42}$ fibrillation process is accelerated with shorter $\tau$ by 
smooth particles at low particle concentrations $(C,<108$ particles/ $\mu \mathrm{L})$, while the elongation rate remains fairly constant. However, with the increase of $C$ up to 144 particles/ $\mu \mathrm{L}$, the fibrillation process is retarded with longer $\tau$. With the further increase of $C$ up to 180 particles $/ \mu \mathrm{L}$, no detectable change of fluorescence intensity is observed during our experimental time window, implying that the fibrillation process is highly suppressed. These observations are consistent with other systems where hydrophobic polymer surfaces were found to influence protein and peptide fibrillation processes, which can be explained based on the interfacial effect on peptide fibrillation. Basically, the hydrophobic PS particle surfaces serve to physically absorb peptide molecules, and such confinement of the two-dimensional environment can locally concentrate peptide molecules and promote the inter-peptide association. The folding of peptide molecules and association of inter-peptide chains can lower the energy barrier into an even more stable landscape, i.e., the formation of critical nuclei, thus accelerating fibrillation processes. Based on this surface-initiated mechanism, the fibrillation kinetics are obviously proportional to the total surface area provided by the PS particles. In the range of low particle surface area $\left(<37 \mathrm{~mm}^{2} / \mathrm{mL}\right.$ corresponding to the particle concentration of 108 particles $/ \mu \mathrm{L}$ ) as shown in Fig. S6b, the interfacial effect on accelerating $\mathrm{A} \beta_{42}$ fibrillation becomes more and more dominate on higher surface area by increasing PS particle concentrations. This is because higher surface area will increase the numbers of peptide molecules on the particle surface, and thus the surface-initiated nucleation of peptides will proceed faster. However, when the total particle surface area is higher than $50 \mathrm{~mm}^{2} / \mathrm{mL}(144$ particles $/ \mu \mathrm{L})$, the $\mathrm{A} \beta_{42}$ fibrillation will be retarded or even inhibited at 180 particles $/ \mu \mathrm{L}$ during the experimental time window. The reason for this can be ascribed to the reduction of ratio of adsorbed $\mathrm{A} \beta_{42}$ molecules on particle surface to the number of the PS particles and the concentration of free $\mathrm{A} \beta_{42}$ molecules in the suspension, which is not high enough for the nucleation of peptides for fibrillation. Fig. S7 shows $A \beta_{42}$ fibrillation process in 
the presence of PS particles at even lower concentration of 5 particles $/ \mu \mathrm{L}$. The reference condition of fibrillation kinetics in the absence of PS particles was also added. The reference condition was the same during the whole fluorescence experiment, including sample incubation and shaking. This result indicates that the retarding and obstacle effect by rough particles is anticorrelated with the surface area of the adding particles. 